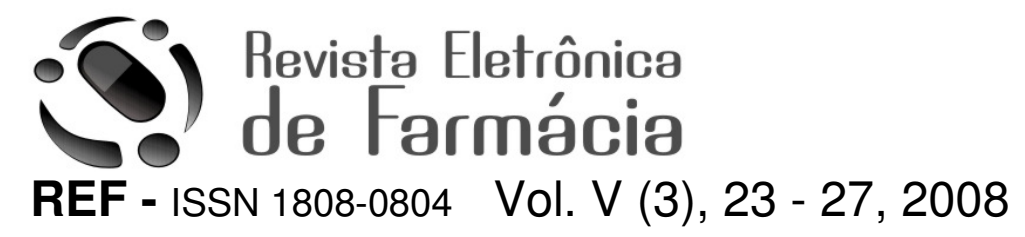

\title{
ESPÉCIES VEGETAIS INDICADAS NO TRATAMENTO DO DIABETES
}

\author{
Vegetal species indicated to use in diabetes treatment
}

\author{
Alzira B. Cecílio ${ }^{1}$, Larissa B. Resende ${ }^{2}$, Aline C. Costa ${ }^{2}$, Mariana M. Cotta ${ }^{2}$, \\ Larissa F. Giacomini ${ }^{2}$, Luíza C. Gomes ${ }^{2}$, Larissa A. Silva ${ }^{2}$, Caroline P.O. Vaz ${ }^{2}$, \\ Francielda Q. Oliveira².
}

\begin{abstract}
${ }^{1}$ Fundação Ezequiel Dias, Laboratório de Virologia Molecular e Bioprodutos, Rua Conde Pereira Carneiro, 80, Gameleira, Belo Horizonte, Minas Gerais, ${ }^{2}$ Centro Universitário Newton Paiva, Laboratório de Farmacobotânica, Av. Silva Lobo, 1730, Nova Granada, Belo Horizonte, Minas Gerais.
\end{abstract}

Autor para correspondência e-mail: alzira@funed.mg.gov.br

Recebido em 21/08/2007 - Aceito em 14/11/2008

RESUMO: Diabetes mellitus é uma desordem crônica causada pelo alto nível de açúcar no sangue. Projeções da OMS demonstram que aproximadamente 150 milhões de pessoas no mundo têm diabetes, sendo que este número provavelmente dobrará até 2025. Várias espécies vêm sendo testadas quanto à sua eficácia no tratamento do diabetes, como por exemplo Taraxacum officinale (dente-de-leão), Cynara scolymus (alcachofra), Arctium lappa (bardana), Baccharis trimera (carqueja), dentre outras. Neste estudo foi possível observar que as 10 plantas mais citadas para esta patologia foram Allium cepa (cebola), Phyllanthus niruri (quebra-pedra), Arctium lappa (bardana), Taraxacum officinale (dente de leão), Syzygium jambolanum (jambolão), Stevia rebaudiana (stévia), Salvia officinalis (sálvia), Eucalyptus globulus (eucalipto), Baccharis trimera (carqueja), Bauhinia forficata (pata- de -vaca). Em relação ao número de espécies por família, a mais representativa foi a FABACEAE (14 espécies); seguida de ASTERACEAE (12 espécies), além das famílias LAMIACEAE (6), MYRTACEAE e ROSACEAE (5), RUTACEAE e APIACEAE (4), SOLANACEAE (3), JUGLANDACEAE, SIMAROUBACEAE, GERANEACEAE, ERICACEAE, BRASSICACEAE, LILIACEAE e EUPHORBIACEAE (2). Os resultados devem contribuir para triagens etnofarmacológicas e direcionamento de pesquisa quanto ao uso de plantas brasileiras para o tratamento de diabetes.

PALAVRAS-CHAVES: diabetes, fitoterapia, tratamento.

\begin{abstract}
Diabetes mellitus is a chronic disorder caused by the elevated level of sugar in blood. Projections of OMS demonstrated that approximately 150 millions of people in the world have diabetes, as this number probably will double untill 2025. Various species have been tested concerning the efficacy diabetes treatment, like Taraxacum officinale (dandelion), Cynara scolymus (globe artichoke), Arctium lappa (burdock), Baccharis trimera (carqueja), among others. In this study it was possible to observe that the most cited plants for this pathology were Allium cepa (onion), Phyllanthus niruri (break stone), Arctium lappa (bardana), Taraxacum officinale (dandelion), Syzygium jambolanum (jambolao), Stevia rebaudiana (stevia), Salvia officinalis (sage), Eucalyptus globulus (eucalyptus), Baccharis trimera (carqueja), Bauhinia forficata (cow's paw). About the number of species concerning each family, the most representative family was FABACEAE (14 species); followed by ASTERACEAE (12 species), and the families LAMIACEAE (6), MYRTACEAE e ROSACEAE (5), RUTACEAE e APIACEAE (4), SOLANACEAE (3), JUGLANDACEAE, SIMAROUBACEAE, GERANEACEAE, ERICACEAE, BRASSICACEAE, LILIACEAE e EUPHORBIACEAE (2). The results might contribute to ethnopharmacological trials and target research towards the use of Brazilian plants in the treatment of diabetes.
\end{abstract}

KEYWORDS: diabetes, phytoterapy, treatment. 
Cecílio, A. B. et al./Revista Eletrônica de Farmácia Vol 5(3), 23 - 28, 2008.

\section{INTRODUÇÃO}

Diabetes mellitus é uma desordem crônica causada relacionada à absorção da glicose do sangue pelas células (CLARE-SALZLER et al., 2003). O diabetes pode aparecer como uma doença auto-imune (tipo I) ou como uma desordem onde a absorção insuficiente da insulina é o principal fator fisio-patológico (tipo II). O diabetes tipo I é conhecido como tipo juvenil e o tipo II como adulto (www.diabetes.org.br). O diabetes pode aparecer sem nenhuma manifestação clínica, anos antes do aparecimento do quadro clínico de diabetes. (FONSECA et al., 2005). Projeções da OMS demonstram que aproximadamente 150 milhões de pessoas no mundo têm o diabetes, sendo que este número provavelmente dobrará até 2025. A demonstração de que esta incidência pode ser reduzida por mudanças do estilo de vida é uma esperança, especialmente para aqueles que possuem familiares próximos com a doença e suas complicações vasculares: perda de visão, insuficiência renal, infarto do miocárdio, derrame cerebral, impotência sexual, dentre outras (WHO, 2005).

Desde tempos imemoriais há relatos do uso das plantas medicinais pelo homem. Baseado no uso popular pesquisas científicas são desenvolvidas para comprovar seus efeitos terapêuticos. Para a utilização segura, os medicamentos fitoterápicos devem cumprir os requisitos básicos de eficácia, segurança e qualidade. Sendo assim, a avaliação dos produtos obtidos a partir de drogas vegetais deve prioritariamente considerar a identificação da droga pela farmacopéia (SHULTZ et al., 2002).

Inúmeras espécies vêm sendo testadas quanto à eficácia no tratamento do diabetes, como por exemplo Taraxacum officinale (dente-de-leão), Cynara scolymus (alcachofra), Arctium lappa (bardana), Baccharis trimera (carqueja), dentre outras espécies (FUENTES et al., 2004;). Diferentes estudos indicam que o alho (Allium sativum L.) contribui na estabilização do nível de açúcar no sangue (BALUCHNEJADMOJARAD \& ROGHANI, 2003). Muitas ervas são conhecidas por afetar o nível de açúcar no sangue, o que pode causar uma significante variação na necessidade de insulina. Na Medicina Tradicional Chinesa, o ginseng tem mostrado bons resultados para regular o nível de açúcar no sangue e é geralmente utilizado para tratar diabetes (XIE et al., 2005).

Este trabalho possui como objetivo a realização de revisão da bibliografia sobre espécies vegetais indicadas no tratamento do diabetes, de maneira a contribuir para triagens etnofarmacológicas e direcionamento de pesquisas do potencial de espécies brasileiras para o tratamento desta patologia.

\section{Matérial e Métodos}

\section{Coleta de dados:}

Para o levantamento bibliográfico foram consultadas referências bibliográficas com informações populares e livros científicos a respeito do uso da fitoterapia para o tratamento do diabetes. Foram consultados 04 livros científicos, 12 livros populares, 07 sites e 19 trabalhos científicos. Com base neste levantamento foi construída uma tabela, onde registrou-se dados sobre as espécies referentes a família, nome científico, nome(s) populares, parte usada, número de citações, citações em que foram encontradas.

\section{Análise dos dados:}

Foram identificadas as doze famílias e 10 espécies mais citadas na bibliografia, sendo estas consideradas em seu potencial para estudos científicos e para uso na terapêutica. Foram realizadas pesquisas de artigos científicos em diferentes bases de dados para verificação de estudos de atividade e toxicidade para as dez plantas mais citadas para o tratamento do diabetes.

\section{RESULTADOS}

Um total de 106 espécies distribuídas em 53 famílias botânicas foram citadas na bibliografia como úteis no tratamento do diabetes. A Tabela 1 contém dados sobre as espécies encontradas no levantamento bibliográfico. As 10 espécies mais citadas na literatura foram Allium cepa (cebola), Phyllanthus niruri ( quebrapedra), Arctium lappa (bardana), Taraxacum officinale (dente de leão), Syzygium jambolanum (jambolão), Stevia rebaudiana (stévia), Salvia officinalis (sálvia), Eucalyptus globulus (eucalipto), Baccharis trimera (carqueja), Bauhinia forficata (pata- de -vaca).

Tabela 1 - Resultado do levantamento das espécies vegetais indicadas na bibliografia para tratamento do diabetes (Anexo 1).

A Tabela 2 contém as dez plantas mais citadas para o tratamento do diabetes. 
Cecílio, A. B. et al./Revista Eletrônica de Farmácia Vol 5(3), 23 - 28, 2008.

Tabela 2- Relação das dez plantas mais citadas para o tratamento do diabetes.

\begin{tabular}{|l|c|}
\hline \multicolumn{1}{|c|}{ (Nome popular/Nome científico/família) } & № de Citações \\
\hline Bardana Arctium lappa L. / ASTERACEAE & 05 \\
\hline $\begin{array}{l}\text { Dente-de-leão Taraxacum officinale Weber ex F.H. Wigg. / } \\
\text { ASTERACEAE }\end{array}$ & 05 \\
\hline Cebola Allium cepa L. / LILIACEAE & 05 \\
\hline Quebra-pedra Phyllanthus niruri L. / EUPHORBIACEAE & 05 \\
\hline Jambolão Syzygium jambolanum L / MYRTACEAE & 05 \\
\hline Stévia Stevia rebaudiana (Bertoni) Bertoni / ASTERACEAE & 05 \\
\hline Sálvia Salvia officinalis L / LAMIACEAE & 06 \\
\hline Eucalipto Eucalyptus globulus Labill / MYRTACEAE & 07 \\
\hline Carqueja Baccharis trimera (Less.) DC. / ASTERACEAE & 09 \\
\hline Pata-de-vaca Bauhinia forficata Link / FABACEAE & 11 \\
\hline
\end{tabular}

Em relação ao número de espécies por família, a mais representativa foi a FABACEAE, com 14 táxons; seguida de ASTERACEAE, com 12 táxons; além das famílias LAMIACEAE com 6 táxons, MYRTACEAE e ROSACEAE com 5 táxons; RUTACEAE e APIACEAE com 4 táxons; SOLANACEAE com 3 táxons; JUGLANDACEAE, SIMAROUBACEAE, GERANEACEAE, ERICACEAE, BRASSICACEAE, LILIACEAE, EUPHORBIACEAE e com 2 táxons..

\section{DISCUSSÃO E CONCLUSÃO}

A Fitoterapia é uma ciência de aplicação para várias patologias. Pesquisas vêm sendo desenvolvidas com o objetivo de comprovar o efeito de espécies vegetais muitas vezes utilizadas apenas baseadas em dados empíricos. O Diabetes mellitus, por ser uma doença crônica, de tratamento contínuo, é um alvo interessante para a busca de novos métodos de tratamento.

Há possibilidade de uso de várias espécies de plantas medicinais para o tratamento do diabetes, contribuindo para triagens etnofarmacológicas e direcionamento de pesquisas do potencial de espécies brasileiras para o tratamento desta condição patológica. As plantas mais citadas nesse trabalho estão incluídas em várias citações em artigos publicados que reforçam o possível uso destas plantas no tratamento do Diabetes mellitus.

A pata-de-vaca (Bauhinia forficata), uma das espécies mais citadas nesta revisão, é usada na medicina popular para o tratamento de diabetes há muito tempo. O decocto pode ser usado no tratamento do diabetes porque melhora a condição do diabetes sem causar toxicidade tecidual detectável. Utilizando-se alguns marcadores apropriados para o modelo experimental em ratos foi administrado diariamente durante sete dias o extrato da planta nas doses de 200 e $400 \mathrm{mg} / \mathrm{kg}$, em ratos diabéticos e não diabéticos. Em conclusão os resultados mostraram que a planta quando administrada, pode reduzir glicose, triglicérides e colesterol total. Estes resultados sugerem a eficácia do uso clínico desta planta no tratamento da diabetes mellitus (PEPATO et al., 2004). Pepato et al., (2002) demonstraram redução significativa da glicose na urina e no soro de ratos tratados com $B$. forficata). $O$ extrato bruto de $B$. candicans apresentou atividade hipoglicêmica com redução da excreção urinária da glicose, sugerindo aumento do metabolismo periferal da glicose (FUENTES et al., 2004).

A carqueja apresenta o segundo maior número de citações para o tratamento do diabetes. A fração aquosa da Baccharis trimera apresentou potencial atividade anti-diabética com redução da glicemia após 7 dias de tratamento quando usada em ratos diabéticos (OLIVEIURA et al., 2005). Em um estudo foi utilizado extratos brutos de carqueja (Baccharis trimera) e Jambolão (Syzygium cumini) a camundongos diabéticos e não diabéticos com tratamento durante sete dias. Nesse estudo, somente frações provenientes de extratos de Baccharis trimera, duas vezes por dia, reduziram a glicemia após tratamento de sete dias. Os resultados sugerem que Baccharis trimera apresentou uma potente atividade anti-diabética (OLIVEIURA et al., 2005). Trabalhos científicos sobre o uso da espécie Syzygium jambolanum não foram encontrados.

O eucalipto (Eucalyptus globulus) foi administrado na forma de decocto à camundongos, e a redução do nível de hiperglicemia pode ser observada (SWANSTON et al., 1990). Experimentos demonstraram a atividade anti-hiperglicêmica do eucalipto, que está associada a estimulação da secreção da insulina, representando um tratamento adicional para o tratamento do diabetes e demonstra potencial para a descoberta de novos compostos ativos para o tratamento do diabetes (GRAY \& FLATT, 1998).

O extrato das folhas da sálvia (Salvia officinalis) apresentou efeito hipoglicemiante em ratos ou camundongos diabéticos e a planta deve ser considerada para pesquisas futuras para uso terapêutico (EIDI et al., 2005; ALARCON-AGUILAR et al., 2002). 
Cecílio, A. B. et al./Revista Eletrônica de Farmácia Vol 5(3), 23 - 28, 2008.

O dente-de-leão (Taraxacum officinale) não afetou os parâmetros da homeostasia da glicose em camundongos (níveis de insulina e glicose basal, hipoglicemia insulina induzida, concentração pancreática do pâncreas) (SWANSTON-FLATT et al., 1989).

Da stévia (Stevia rebaudiana) pode ser extraído o steviosídeo (glicosídeo diterpeno) que administrado juntamente com a soja parece exercer um tratamento efetivo na hiperglicemia em ratos. Outros estudos são necessários para a verificação dos resultados obtidos em animais diabéticos possam ser obtidos em humanos (DYRSKOG et al., 2005). O steviosídeo foi capaz de regular a deficiência em insulina em ratos (CHEN et al., 2005) e reduzir a glicose no sangue em pacientes com diabetes tipo 2, indicando efeitos benéficos no metabolismo da glicose (GREGERSEN et al., 2004).

A bardana (Arctium lappa) não apresentou citações científicas nas fontes usadas para este levantamento. Apesar de ser citada popularmente, a ausência de citações científicas sobre o uso da bardana pode estar ligado a ausência de uma ação específica em relação ao Diabetes mellitus e/ou a presença de substâncias ativas em pouca concentração para serem testadas.

Diferentes estudos indicaram que o Allium cepa (cebola) não demonstrou efeito hipoglicemiante no tratamento de ratos diabéticos (JELODAR et al., 2005; EL-DEMERDASH et al., 2005).

Preparações com todas as partes de Phyllanthus niruri ( quebra-pedra) foram administradas por 10 dias para pacientes com diabetes mellitus. Os resultados indicaram que esta planta possui um potencial efeito diurético, hipotensivo e hipoglicemiante para humanos (SRIVIDYA \& PERWAL, 1995).

Interessante notar que a Família Fabaceae foi a que apresentou maior número de espécies citadas, sendo esta família muito importante do ponto de vista medicinal, econômico e a família à qual pertence a Bauhinia forficata, amplamente empregada no tratamento do diabetes e investigada em ensaios in vitro e in vivo.

A Família Asteraceae foi descrita inicialmente como Compositae por Dietrich Giseke. Essa família compreende 1528 gêneros, com aproximadamente 22750 espécies encontradas em todo o planeta, sendo a maior família botânica dentre as angiospermas, considerada uma as maiores fontes de espécies vegetais de interesse terapêutico, dentre as quais encontra-se a Cynara solymus (alcachofra) e Baccharis trimera (carqueja) (DI STASI \& HIRUMA-LIMA, 2002).

A Família Lamiaceae inclui cerca de 252 gêneros, nos quais se distribuem 6700 espécies. Além da importância do ponto de vista medicinal, esta família também é fonte de espécies com grande valor como condimentos, alimentos e na indústria de perfumes e cosméticos (DI STASI \& HIRUMA-LIMA, 2002).

Os resultados podem contribuir para incentivar a pesquisa na busca de novos compostos ativos para o tratamento do Diabetes mellitus. São necessários maiores investimentos para estudos científicos nessa área, de maneira a levar à comprovação da eficácia dessas espécies como hipoglicemiantes.

\section{REFERÊNCIAS BIBLIOGRÁFICAS}

ALARCON-AGUILAR FJ, ROMAN-RAMOS R, FLORES-SAENZ JL, AGUIRRE-GARCIA F. Investigation on the hypoglycaemic effects of extracts of four mexican medicinal plants in normal and alloxan-diabetic mice. Phytother Res. v.16, n.4, p.383-6,2002.

Aquimia Vilabol. Plantas Medicinais. http://www.aquimia.vilabol.uol.com.Br/plantasmedicinais/page5.html (capturado em 07 de setembro 2005).

BALBACH A. As Frutas na Medicina Doméstica. Itaquaquecetuba: Editora Missionária, 1992 a.

BALBACH A. As Hortaliças na Medicina Doméstica. Itaquaquecetuba: Editora Missionária, 1992 b.

BALBACH A. As Plantas Curam. São Paulo: Vida Plena, 1993.

BALUCHNEJADMOJARAD T, ROGHANI M. Edothelium-dependent and independent effect of aqueous extract of garli on vascular reactivity on diabetic rats. Fitoterapia. v.74. n.7-8, p. 630-7, 2003.

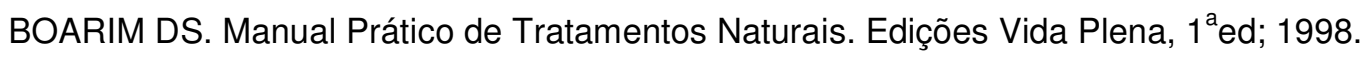

BRANDÃO MGL. Circuito das Plantas Medicinais e Aromáticas de Belo Horizonte.

CHEN TH, CHEN SC, CHAN P, CHU YL, YANG HY, CHENG JT. Mechanism of the hypoglycemic effects of stevioside, a glycoside of Stevia rebaudiana. Planta Med. v.71. n.2, p.108-13, 2005.

CLARE-SALZLER MJ, CRAWFORD JM, KUMAR V. The Pancreas. In: Kumar V, Cotran RS, Robbins SL, eds. Basic Pathology $7^{a} \mathrm{e} e d$. Filadélfia: Editora Saunders, 2003. p.635-655. 
Cecílio, A. B. et al./Revista Eletrônica de Farmácia Vol 5(3), 23 - 28, 2008.

CORREAA AD, BATISTA RS, QUINTAS LEM. Plantas medicinais: do cultivo à terapêutica. Petrópolis: Editora Vozes, 4.ed; 2001.

DI STASI LC, HIRUMA-LIMA CA. Plantas medicinais na Amazônia e na Mata Atlântica, $2^{2}$ ed. São Paulo: Editora Unesp, 2002. 605p.

DYRSKOG SE, JEPPESEN PB, COLOMBO M, ABUDULA R, HERMANSEN K. Preventive effects of a soy-based diet supplemented with stevioside onthe development of the metabolic syndrome and type 2 diabetes in Zucker diabetic fatty rats. Metabolism. v.54, n.9, p.1181-8, 2005.

Educa Terra. Plantas Medicinais. http://www.educaterraterra.com.br/almanaque/ciencia/plantas_medicinaisZ (acessado em 07/Set/2005).

EIDI M, EIDI A, ZAMANIZADEH H. Effect of Salvi alis L. Leaves on serum glucose and insulin in healthy and streptozotocin-induced diabetis rats. J Ethnopharmacol. v.100, n.3, p.310-3, 2005.

EL-DEMERDASH FM, YOUSEF MI, EL-NAGA NI. Biochemical study on the hypoglycemic effects of onion and garlic in alloxan-induced diabetic rats. Food Chem Toxicol. v.43, n.1, p.57-63, 2005.

FETROW CW, ÁVILAJR. Manual de medicina alternativa para o profissional. Rio de Janeiro: Editora Guanabara Kooga; 2000.

FONSECA CT, AMARAL DM, RIBEIRO, MG, BESERRA IC, GUIMARAES MM. Insulin resistance in adolescents with Down syndrome: a cross-sectional study. BMC Endocr Disord. v.17, p. 5-6, 2005.

FRANCOIJ, FONTANA UL. Ervas e Plantas: A Medicina dos Simples. Erexim,R.S.: Editora Edebra, $7^{\mathrm{a}}$ ed; 2002.

FUENTES O, ARANCIBIA-AVILA P, ALARCON J. Hypoglycemic activity of Bauhinia candicans in diabetic induced rabbits. Fitoterapia. v.75, n.6, p.527-32, 2004.

FUENTES O, ARANCIBA-AVILA P, ALARCON J. Hypoglycemic activity of Bauhinia candicans in diabetic induced rabbits. Fitoterapia. v. 75, n.6, p.527-32, 2004.

GRAY AM, FLATT PR. Antihyperglycemic actions of Eucalyptus globulus (Eucalyptus) are associated with pancreatic and extra-pancreatic effects in mice. Bioch Mol Roles of Nutrients. p.2319-2323, 1998.

GREGERSEN S, JEPPESEN PB, HOLST JJ, HERMANSEN K. Antihyperglycemic effects of stevioside in type 2 diabetic subjects. Metabolism. v.53, n.1, p.73-6, 2004.

JELODAR GA, MALEKI M, MOTADAYEN MH, SIRUS S. Effect of fenugreek, onion and garlic on boold glucose and histopathology of pancreas of alloxan-induced diabetic rats. Indian J Med Sci; v.59, n.2, p.64-9, 2005.

Linguagem das Flores. Produtos Biológicos. http://linguagemdasflores.blogs.sapo.pt/arquivo/2004_04.html (acessado em 07/Set/2005).

MARTINS ER. Plantas Medicinais. Viçosa: Editora UFV; 2000.

MATOS FJA. Farmácias vivas: sistema de utilização de plantas medicinais projetado para pequenas comunidades. Fortaleza: Editora UFC, 3. ed.; 1998.

Minuto Poético. Plantas Medicinais. http:// www.minuto.poetico.nom.br (acessado em 07/Set/2005).

OLIVEIURA AC, ENDRINGER DC, AMORIM LA, BRANDAO MD, COELHO MM. Effect of the extracts and fractions of Baccharis trimera and Syzygium cumini on glycaemia of diabetic and non-diabetic mice. $J$ Ethnopharmacol . v.28, (epub ahead of print), 2005.

PEPATO MT, KELLER EH, BAVIERA AM, KETTELHUT IC, VENDRAMINI RC, BRUNETTI IL. Anti-diabetic activity of Bauhinia forficata decoction in stretozotocin-diabetic rats. J Ethnopharmacol. v.81, n.2, p.191-7, 2002.

PEPATO MT, BAVIERA AM, VENDRAMINI RC, BRUNETTI IL. Evaluation of toxicity after one-months treatement with Bauhinia forficata decoction in stretozotocin-induced diabetic rats. BMC Complementary and Alternative Medicine. v.4, p.1-7, 2004. 


\section{Cecílio, A. B. et al./Revista Eletrônica de Farmácia Vol 5(3), 23 - 28, 2008.}

RioNet. Canto Verde. http://www.rionet.com.br/ cantoverde/p.html (acessado em 07/Set/2005).

RIQUEIRO MP. Plantas que Curam: Manual Ilustrativo de Plantas Medicinais. Editora: Paulus, 6 a ed; 1992.

SCHULTZ, V, HÄNSEL R, TYLER VE. Fitoterapia racional: um guia de fitoterapia para as ciências da saúde. São Paulo:Editora Manole, 2002; p.386.

SPETHMANN CN. Medicina Altenativa de A a Z. Editora Natureza, $7^{\mathrm{a}}$ edição; Setembro de 2004.

SRIVIDYA N, PERIWAL S. Diuretic, hypotensive and hypoglycaemic effect of Phyllanthus amarus. Indian J Exp Biol . v.33, n.11, p.861-4, 1995.

SWANSTON-FLATT SK, DAY C, FLATT PR, GOULD BJ, BAILEY CJ. Glycaemic effects of tradional european plant treatments for diabetes, studies in normal and streptozocin diabetic mice. Diabetes Res. v.10, n.2, p.69-73, 1989.

SWANSTON-FLATT SK, DAY C, BAILEY CJ, FLATT PR. Traditional plant treatements for diabetes. Studies in normal and streptozotocin diabetic mice. Diabetologia. v.33, n.8, p.462-4, 1990.

USP. Plantas Medicinais, Aromáticas e Condimentares. http://www.ci-66.ciagri.usp.br/pm/index.asp(acessad 07/Set/2005).

WILLIANS, T. Fitoterapia: Guia Prático. Editora: Callis Ltda; 1998.

World Health Organization. Diabetes: the cost of diabetes. http://www.who.int/mediacentre/factsheets/fs138/en/ (acessado em 07/Set/2005).

XIE JT, WANG CZ, WANG AB, WU J, BASILA D, YUAN CS. Antihyperglycemic effects of total ginsenosides from leaves and stem of Panax ginseng.

Acta Pharmacol Sin. v.26, n.9, p.1104-10, 2005.

YWATA C, ANTÔNIO J, CORDEIRO R. Medicina Natural - A Cura está na Natureza. Editora Três. 